\title{
Selenium and Polycystic Ovary Syndrome; Current Knowledge and Future Directions: A Systematic Review
}

Authors

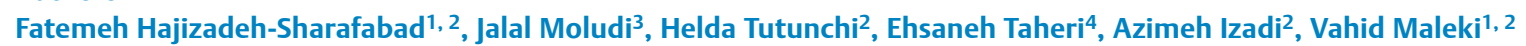

Affiliations

1 Student Research Committee, Tabriz University of Medical Sciences, Tabriz, Iran

2 Nutrition Research Center, Faculty of Nutrition and Food Sciences, Tabriz University of Medical Sciences, Tabriz, Iran

3 Department of Nutrition, Faculty of Nutrition Sciences and Food Technology, Kermanshah University of Medical Sciences, Kermanshah, Iran

4 Nutrition and Endocrine Research Center, Research Institute for Endocrine Sciences, Shahid Beheshti University of Medical Sciences, Tehran, Iran

Key words

selenium, PCOS, insulin resistance, dyslipidemia

$$
\begin{array}{ll}
\text { received } & 11.02 .2019 \\
\text { accepted } & 02.04 .2019
\end{array}
$$

\section{Bibliography}

DOI https://doi.org/10.1055/a-0890-6823

Horm Metab Res 2019; 51: 279-287

(c) Georg Thieme Verlag KG Stuttgart · New York

ISSN 0018-5043

Correspondence

Vahid Maleki

Department of Clinical Nutrition,

Faculty of Nutrition,

Tabriz University of Medical Sciences,

Ghol-Ghashtst,

Tabriz 5166614711,

I. R.

Iran

Tel.: + 98/935/7097 463, Fax: + 98/413/3340634

malekivahid433@yahoo.com

\section{ABSTRACT}

Polycystic ovary syndrome (PCOS), as the most common endocrine disorder in reproductive-aged women, is recognized by hyperandrogenism and insulin resistance. Selenium (Se) potentially possesses therapeutic effects on PCOS due to antioxidant and insulin-like properties. This systematic review evaluates the potential role of Se in the complications of PCOS. A systematic review was performed on published studies reporting the effects of Se on PCOS. Three major databases including PubMed, Scopus, and Google Scholar were searched until December 2018. A total of 7 human studies and two in vitro studies met the inclusion criteria. Two out of three case-control studies showed that serum Se levels tend to decrease in patients with PCOS. Of four studies that evaluated the impact of Se supplementation on insulin resistance, only one study showed protective effects of Se against insulin resistance. Two out of three studies reported the antioxidant effect of Se. Few studies investigating anti-androgenic effect of Se presented controversial results. There were three studies that evaluated the anti-hyperlipidemic effect of Se, of which two surveys indicated the lowering effects of Se on VLDL and LDL-cholesterol. The reviewed studies confirmed inverse relationships between serum Se levels and some androgenic hormones in PCOS. Se is able to attenuate insulin resistance and dyslipidemia. The available data are currently insufficient to support the protective effects of Se on PCOS.

\section{Introduction}

Polycystic ovary syndrome (PCOS) is known as one of the most common endocrine disorder in women of reproductive age worldwide [1]. There is variation in the prevalence of PCOS widely depending on ethnicity, body composition, and the definition used for its diagnosis. However, it is estimated that approximately $2-20 \%$ of reproductive-aged women are suffering from PCOS [2].
PCOS is identified by symptoms of hyperandrogenism, obesity, amenorrhea, hirsutism, acne, androgenic alopecia, and biochemical status of raised androgens such as testosterone with irregular menses and infertility [3]. Although the exact cause of PCOS is still uncertain, it seems that involved factors are genetic, environmental, and unhealthy lifestyle [4]. The main key to the pathogenesis of PCOS is hyperandrogenism and insulin resistance ( $\bullet$ Fig. 1). Metabolic abnormalities are associated with PCOS, including insulin 


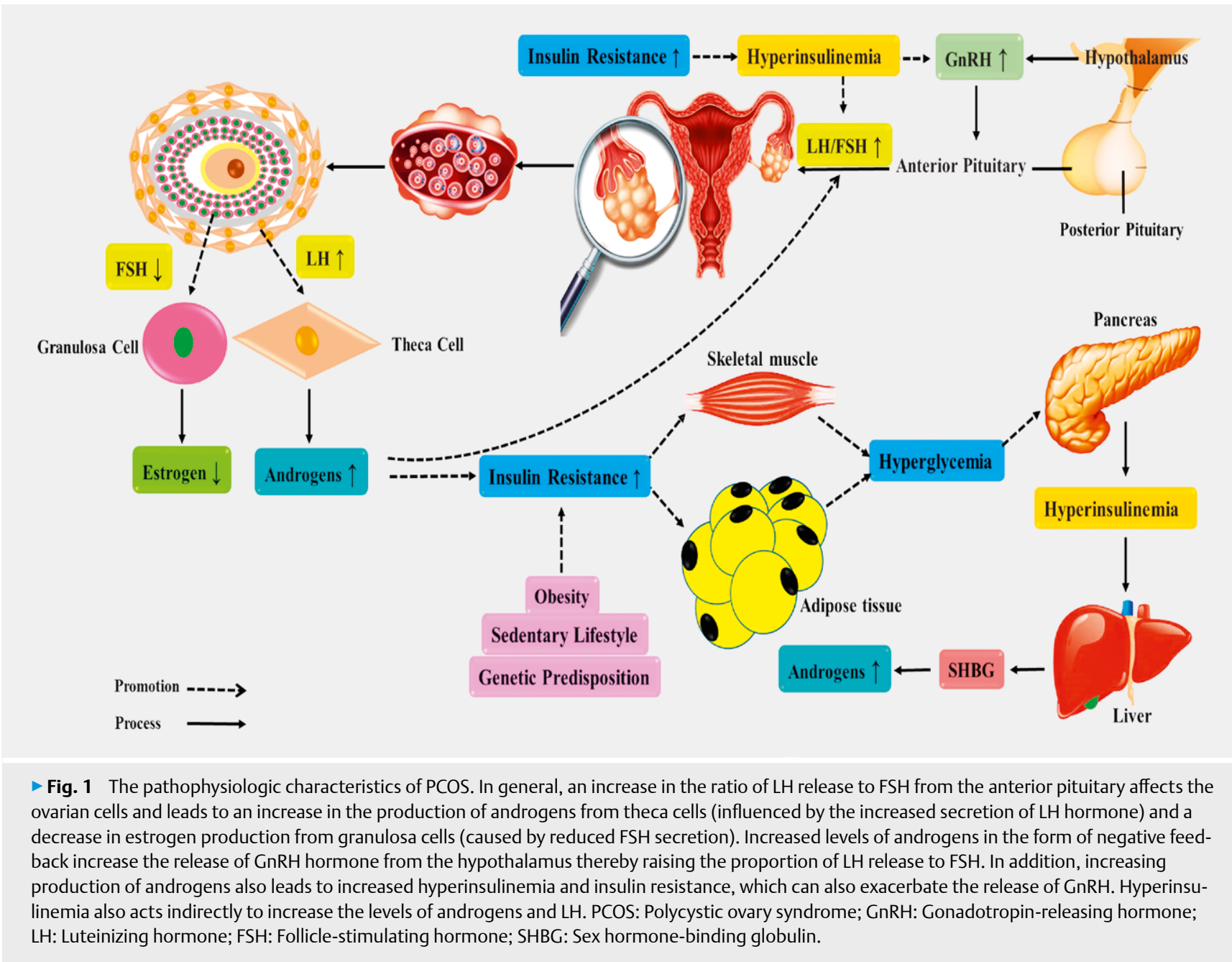

resistance, obesity, type 2 diabetes, cardiovascular disease, dyslipidemia, inflammation, and stress oxidative, which are common in PCOS [5]. Increasing androgen, obesity, insulin resistance, and related disorders can lead to increased inflammation and oxidative stress [6].

Nowadays, the use of complementary therapy and alternatives in medicine is known as a good strategy to improve hyperandrogenism and its associated degenerative effects on PCOS [7]. Selenium (Se) is an indispensable trace element for humans because of its antioxidant and anti-inflammatory properties [8]. It is a trace element that is important for the maintenance of human health, which operates as an integral part of selenoproteins assisting redox processes as an effective antioxidant [9]. The many beneficial roles of supplementation with Se in improving reproductive outcomes, inflammatory biomarkers, and oxidative stress may be linked to its inhibitory effects on pro-inflammatory cytokines and reactive oxygen and nitrogen species [10]. However, clinical conditions of PCOS might indirectly affect Se status in the context of their pro-inflammatory effects and thus change hormonal parameters and actions as well [11]. Therefore, Se is assumed to likely affect the reproductive status, inflammatory factors, and oxidative stress biomarkers in PCOS.

The present systematic review aims to explore the literature on the effect and status of Se regarding weight changes, glycemic state, dyslipidemia, ovarian hormones, androgen levels, biomarkers of inflammation, and oxidative stress in PCOS. Furthermore, exploring the knowledge gaps are addressed and recommendations are provided for future research.

\section{Materials and Methods}

\section{Search strategy}

To explore studies related to this systematic review, PubMed, Scopus, and Google Scholar were searched from inception to December 2018 using keywords such as "selenium" or "selenate" or "selenite" or "selenomethionine" or "selenocysteine" or "ebselen" or "selenious acid" or "selenium-binding proteins" AND "polycystic ovary syndrome" or "PCOS" or "sclerocystic ovary syndrome" or "dysmetabolic syndrome". All articles published in English-language journals were eligible. This systematic review was performed based on the PRISMA guideline and its protocol is registered with the PROSPERO database of systematic reviews (registration number: CRD42018083239). 


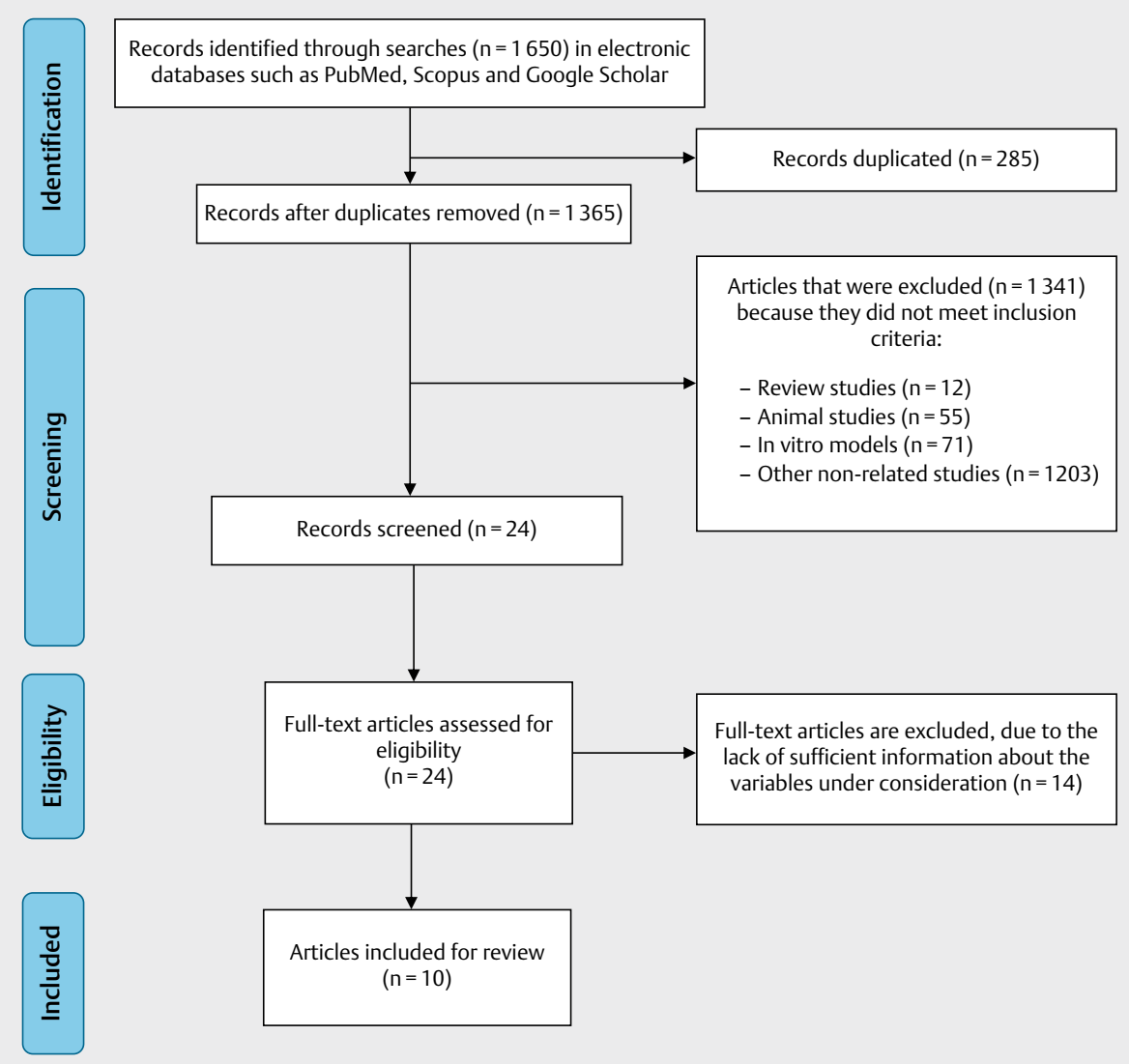

- Fig. 2 Flowchart of the process for selecting studies for the systematic review.

\section{Inclusion and exclusion criteria}

The titles and abstracts of all studies were searched by two researchers and reviewed independently. All in vivo and in vitro studies that examined the role of Se in PCOS and were published in English-language journals were eligible in this review. Those with insufficient data on pregnant women, letters, comments, and ecological studies were excluded.

\section{Quality assessment of studies}

At first, full texts of all studies that met inclusion criteria were evaluated based on the checklist of the objectives and research questions. Then the information was extracted by the researcher separately. Finally, the accuracy of the data obtained was examined by a third researcher.

\section{Data extraction}

Two reviewers independently screened the data of eligible full-text articles based on the characteristics of the study including location and design of the study, information of the participants, and the main outcome. Any disagreement between the reviewers was resolved by discussion between the researchers.

\section{Findings}

Totally, 1650 potentially eligible studies were retrieved herein, of which 285 cases were duplicated publication, leading to 1365 non-duplicated publications. Of these, 24 studies were excluded because they did not meet the inclusion criteria, and one study was omitted due to lack of adequate information on the variables under examination. Finally, 10 articles met the inclusion criteria. A summarized flowchart of the selection process of studies for the systematic review is shown in $>$ Fig. 2.

\section{Study characteristics}

Characteristics and the main outcomes of the 10 articles [12-21] included in the systematic review are summarized in $\triangleright$ Table 1 . The trials were published between 2013 and 2018. Of all the identified studies, six studies were conducted in Iran. five studies [12, 16, $19,20,22]$ used a randomized clinical trial design, whereas the others used a case-control design. All of the five trials used $200 \mu \mathrm{g} \mathrm{Se}$ orally.

\section{Selenium and weight changes in PCOS}

In the long term, obesity is considered a risk factor for infertility, PCOS, dyslipidemia, and cardiovascular disease risk factors [23]. It has been reported that subjects with obesity have a greater risk for PCOS, which is more likely to affect the severity of PCOS [23, 24].

There is some evidence proposing that Se supplementation decreases the BMI and weight of PCOS patients. For instance, in women with PCOS, it was shown that co-supplementation with $200 \mu \mathrm{g} /$ day of Se plus $8 \times 10^{9} \mathrm{CFU} /$ day probiotic for 12 weeks de- 
- Table 1 Characteristics of studies that reported the roles of selenium in PCOS.

\begin{tabular}{|c|c|c|c|c|}
\hline $\begin{array}{l}\text { Type of } \\
\text { study }\end{array}$ & Authors/date & Source & Model & Results (treatment group) \\
\hline \multirow[t]{2}{*}{ In vitro } & $\begin{array}{l}\text { Montes-Nieto et al. } \\
2013 \text { [14] }\end{array}$ & Spain & $\begin{array}{l}\text { Subcutaneous (SAT) and visceral (VAT) adipose } \\
\text { tissue samples from patients with bariatric surgery }\end{array}$ & - SBP1 increased in VAT compared with SAT \\
\hline & $\begin{array}{l}\text { Köse et al. } \\
2014[21]\end{array}$ & Turkey & $\begin{array}{l}\text { Neutrophils from patients with PCOS treated } \\
\text { with Se }\end{array}$ & $\begin{array}{l}\text { - Antioxidant capacity increased } \\
\text { - Lipid peroxidation levels decreased }\end{array}$ \\
\hline \multirow[t]{8}{*}{ Human } & $\begin{array}{l}\text { Razavi et al. } \\
2016[22]\end{array}$ & Iran & $\begin{array}{l}\text { Women aged } 18-40 \text { years old with PCOS } \\
\text { received } 200 \mu \mathrm{g} / \text { day Se as a Se-enriched yeast } \\
\text { tablet or placebo for } 8 \text { weeks }\end{array}$ & $\begin{array}{l}\text { - Pregnancy rate increased } \\
\text { - Acne and hirsutism decreased } \\
\text { - DHEA, hs-CRP, and plasma MDA levels decreased } \\
\text { - No significant difference in the hormonal profiles, } \\
\text { NO and other biomarkers of oxidative stress }\end{array}$ \\
\hline & $\begin{array}{l}\text { Jamilian et al. } \\
2014[20]\end{array}$ & Iran & $\begin{array}{l}18-40 \text { years old PCOS women orally supplement- } \\
\text { ed with } 200 \mu \mathrm{g} / \text { day Se for } 8 \text { weeks. }\end{array}$ & $\begin{array}{l}\text { - Insulin levels, HOMA-IR and lipid profile improved } \\
\text { - No affection on FPG } \\
\text { - DHEA, hs-CRP, and plasma MDA levels decreased }\end{array}$ \\
\hline & $\begin{array}{l}\text { Hosseinzadeh et al. } \\
2016[19]\end{array}$ & Iran & $\begin{array}{l}\text { Daily administration of } 200 \mu \mathrm{g} \text { Se as a Se-en- } \\
\text { riched yeast tablet in PCOS patients aged 18-42 } \\
\text { years old for } 12 \text { weeks }\end{array}$ & $\begin{array}{l}\text { - Insulin resistance increased } \\
\text { - No significant changes in glucose, total testoster- } \\
\text { one, SHBG and FAI }\end{array}$ \\
\hline & $\begin{array}{l}\text { Coskun et al. } \\
2013[13]\end{array}$ & Turkey & $\begin{array}{l}\text { Se levels and their relation with insulin resistance } \\
\text { and hyperandrogenism in PCOS patients } \\
\text { compered healthy cases }\end{array}$ & $\begin{array}{l}\text { PCOS group had: } \\
\text { - Lower serum levels of Se, } \\
\text { - Lower serum levels of FSH, } \\
\text { - Higher serum levels of LH, E2, and DHEAS, } \\
\text { - No significant difference in the insulin levels and } \\
\text { HOMA-IR, compared to the control group }\end{array}$ \\
\hline & $\begin{array}{l}\text { Kanafchian et al. } \\
2017[18]\end{array}$ & Iran & $\begin{array}{l}\text { Case control study design to evaluated serum Se } \\
\text { and their relationship with insulin resistance in } \\
\text { PCOS patients }\end{array}$ & $\begin{array}{l}\text { PCOS group had: } \\
\text { - Lower serum levels of Se, } \\
\text { - No significant relationship between Se levels and } \\
\text { insulin resistance, Compared to the control group }\end{array}$ \\
\hline & $\begin{array}{l}\text { Zagrodzki et al. } \\
2017[17]\end{array}$ & Poland & $\begin{array}{l}\text { In case control study, Se status were compared in } \\
\text { patients with PCOS }\end{array}$ & $\begin{array}{l}\text { PCOS group had: } \\
\text { - No apparent differences in Se levels, } \\
\text { - No significant relationship between GPX3 and } \\
\text { SELENOP, } \\
\text { - A significant correlation between serum Se with } \\
\text { GPX3 and SELENOP levels, Compared to the } \\
\text { control group }\end{array}$ \\
\hline & $\begin{array}{l}\text { Modarres et al. } \\
2017[16]\end{array}$ & Iran & $\begin{array}{l}\text { Se supplementation ( } 200 \mu \mathrm{g} / \text { day as a Se-enriched } \\
\text { yeast tablet) for } 8 \text { weeks on gene expression } \\
\text { related to insulin and lipid in infertile women aged } \\
18-40 \text { years old with PCOS candidate for IVF }\end{array}$ & $\begin{array}{l}\text { - Gene expression levels of LDLR decreased } \\
\text { - Gene expression levels of PPAR- } \gamma \text { and GLUT-1 } \\
\text { increased } \\
\text { - No affection on LP (a) }\end{array}$ \\
\hline & $\begin{array}{l}\text { Shabani et al. } \\
2018 \text { [12] }\end{array}$ & Iran & $\begin{array}{l}200 \mu \mathrm{g} / \text { day Se plus } 8 \times 10^{9} \mathrm{CFU} / \text { day probiotic } \\
\text { co-supplementation for } 12 \text { weeks on weight loss, } \\
\text { glycemic control and markers of cardio-metabol- } \\
\text { ic risk in women aged } 18-40 \text { years old with PCOS }\end{array}$ & $\begin{array}{l}\text { - Weight, insulin resistance and markers of } \\
\text { cardio-metabolic risk decreased } \\
\text { - No affection on FBG and HDL-cholesterol levels }\end{array}$ \\
\hline
\end{tabular}

DHEA: Dehydroepiandrosterone; FAI: Free androgen index; FPG: Fasting plasma glucose; hs-CRP: High sensitivity C-reactive protein; HOMA-IR: Homeostasis model of assessment-insulin resistance; MDA: Malondialdehyde; LDL: Low-density lipoprotein; HDL: High-density lipoprotein; VLDL: Very low-density lipoprotein; IR: insulin resistance; SBP1: Se-binding protein 1; SELENOP: Plasma selenoprotein P; SHBG: Sex hormone binding globulin; PPAR-Y: Peroxisome proliferator-activated receptor gamma; LDLR: Low-density lipoprotein receptor; GLUT-1: Glucose transporter 1; GPX3: Glutathione peroxidase; LP (a): Lipoprotein(a); NO: Nitric oxide; IVF: In vitro fertilization.

creased body weight and markers of cardio-metabolic risk [12]. However, another study reported that supplementation with $200 \mu \mathrm{g}$ of Se daily for 8 weeks did not significantly affect the BMI and weight [22]. In line with some previous studies [16, 20], Hosseinzadeh et al. indicated that women with PCOS supplied with oral administration of $200 \mu \mathrm{g} /$ day of Se for 12 weeks failed to observe any weight lowering effect [19]. The discrepancy in the find- ings may be due to differences in the duration of intervention, dietary intake, physical activity level, and the clinical state of the participants. In addition, Se may reduce both weight and BMI through affecting insulin concentrations, which in turn reduces insulin-like growth factors (IGFs) and their binding proteins [20, 25]. These findings encourage further research on the promising role of Se in the weight loss of patients with PCOS. 


\section{Selenium and glycemic control in PCOS}

Insulin resistance and hyperglycemia are the most prominent features of patients with PCOS $[20,25]$. Insulin resistance is the most common metabolic disorder in PCOS, with approximately more than $50 \%$ of patients with PCOS having borderline or high insulin levels $[19,26]$.

There are many studies with controversial results on the effects of Se on glucose homeostasis as the most common metabolic disorder in PCOS, with approximately more than $50 \%$ of patients with PCOS having borderline or high insulin levels. Beneficial effects of Se on improved glycemic control might result from its effect on significantly increased gene expression levels of peroxisome proliferator-activated receptor gamma (PPAR- $\gamma$ ) and glucose transporter 1 (GLUT-1) [16]. It also affects several essential functions including insulin-like action, thyroid hormone metabolism, protection from redox homeostasis and oxidative stress [8]. Some previous studies on PCOS indicated that PCOS women had significantly low levels of plasma Se in comparison to healthy women [19]. On the contrary, a recent study [18] on blood trace element concentrations in PCOS subjects demonstrated that there were no differences in serum Se levels of these patients compared to that of the control group.

A study by Coskun et al. [13] revealed that although women with PCOS had lower serum levels of Se, no relationships were observed between blood glucose and insulin levels with blood Se concentrations. The following studies were conducted to investigate the potential role of selenium on glycemic status in patients with PCOS.

In a study by Jamilian et al. [20], it was observed that oral supplementation of $200 \mu \mathrm{g} /$ day of Se for 8 weeks led to a significant improvement in insulin sensitivity characterized by an increase in quantitative insulin sensitivity check index (QUICKI) and decreases in homeostasis model of assessment-insulin resistance (HOMA-IR) and serum insulin levels. Similarly, Shabani et al. [12] stated that $200 \mu \mathrm{g} /$ day Se plus $8 \times 10$ CFU/day probiotic supplements in women with PCOS had beneficial effects on improved insulin metabolism, but did not affect FPG levels. On the contrary, Hosseinzadeh et al. [19] indicated that administration of $200 \mu \mathrm{g}$ of Se as a Se-enriched yeast tablet for 12 weeks in women with PCOS did not yield any effect on fasting blood sugar (FBS). Also, they concluded that 12 weeks of Se supplementation increased insulin resistance in PCOS patients. Therefore, until the results of additional investigations become available, the use of Se supplements in these patients need to warrant more caution. Similarly, Shabani [12] and Jamilian [20] did not observe any effect of Se supplement on FBG levels. Some investigators, however, indicated that a 3-month Se supplementation in subjects with T2DM significantly decreased fasting plasma glucose (FPG) levels indicating that Se supplementation may lead to reduced insulin resistance. Moreover, Se supplementation was reported to have a good effect on lowering the FBS, though, it was not significant $[12,20]$. There is nearly a general agreement that Se may stimulate the insulin cell signaling pathway, resulting in a diminished insulin resistance $[12,16,20]$.

\section{Selenium and dyslipidemia in PCOS}

Dyslipidemia is one of the common metabolic disorders due to hormonal changes and insulin resistance in patients with PCOS, which is one of the most important risk factors for atherosclerosis [27, 28]. Also, dyslipidemia in PCOS women contributes to reproductive dis- orders. In addition, insulin resistance and hyperandrogenism have been observed in PCOS women, therefore, they are more likely to develop an intrinsic lipid abnormality [29]. Increased gene expression levels of some enzymes such as lipoprotein (a) [LP (a)], low-density lipoprotein receptor (LDLR), and $\beta$-oxidation following Se supplementation may improve lipid metabolism [30].

Previous studies reported diverse effects of Se on lipid profile. Shabani et al. [12] indicated that $200 \mu \mathrm{g} /$ day of Se supplementation for 12 weeks led to significant decreases in LDL, TG, and TC and improvement of HDL in women with PCOS; however, no changes were observed in PCOS patients, indicating that Se supplementation did not affect TG. Also, Jamilian et al. [20] found that after 8 weeks of intervention with Se, no differences were observed in term of HDL and HDL, though, it decreased TG levels. The different findings may be due to differences in the baseline lipid profile, dietary intake, and blood Se levels of the participants. Overall, Se may have a possible beneficial impact on dyslipidemia, yet, related studies are scarce and more investigations are needed to find out the protective effects of Se on lipid profile.

\section{Selenium and ovarian hormones and androgens in PCOS}

Previous studies have demonstrated that PCOS is associated with female infertility, which is characterized by hirsutism, hyperandrogenism, anovulation, and menstrual disturbance [13, 31]. In addition, insulin resistance is also responsible for increased hyperinsulinism, which in turn contributes to the production of high androgen levels and increased ratio of LH to FSH [32-34].

In this regard, previous studies showed that Se might affect several reproductive and obstetric complications including infertility. Razavi et al. [22] reported that $200 \mu \mathrm{g}$ of Se daily for 8 weeks had a beneficial effect on reproductive outcomes, especially on decreasing serum dehydroepiandrosterone (DHEA) levels in PCOS subjects. Additionally, patients who received Se supplements had significantly higher pregnancy rates and decreased acne and/or hirsutism compared with placebo-fed subjects [22]. However, Hosseinzadeh et al. showed that administration of $200 \mu \mathrm{g}$ of Se supplements for 12 weeks did not lead to significant changes in hormonal profiles including total testosterone and SHBG concentrations [19]. It should be noted that there is such a paucity of data on the effect of Se supplements on obstetric complications. Furthermore, carefully designed studies are needed to evaluate the effect of Se in this context.

\section{Selenium and biomarkers of inflammation, and oxidative stress in PCOS}

Increased levels of pro-inflammatory cytokines and oxidative stress markers have been reported in patients with PCOS [35, 36]. Likewise, previous studies demonstrated that women with PCOS presented significantly lower total antioxidant capacity (TAC) levels in comparison with controls $[37,38]$. On the other hand, these markers of oxidative stress are negatively associated with testosterone in women with PCOS [37]. Oxidative stress and low grade inflammation play a fundamental role in infertility, which also impairs insulin action [36, 39]. All of these could together lead to increased cardio-metabolic risk in these women [40]. 
Beneficial effects of Se supplementation on improved oxidative stress may result from its important role in redox reactions, such as glutathione peroxidase (GPX) and superoxide dismutase (SOD) $[41,42]$. In addition, Se might decrease hydroperoxides levels via preventing the production of proinflammatory cytokines including TNF- $\alpha$ and IL-1 [43]. Few studies have reported the role of Se supplementation on oxidative stress and inflammation in PCOS women. For instance, Razavi et al. [22] indicated that Se supplementation in women with PCOS led to a significant improvement in serum high sensitivity C-reactive protein (hs-CRP), and plasma malondialdehyde (MDA) levels, but it did not influence nitric oxide (NO) and other biomarkers of oxidative stress. Se appears to provide a protective effect against oxidative stress and Ca entry through modulation of neutrophil TRP vanilloid 1 (TRPV1) calcium channels [21]. Köse reported that antioxidant effects of Se on the modulation of neutrophil TRPV1 calcium channels decreased lipid peroxidation levels in neutrophils from PCOS patients [21].

\section{Discussion}

Based on the findings of the present systematic review on 10 studies [12-21], Se supplementation shows remarkable effects on PCOS patients. In spite of substantial heterogeneity in studies, Se may have modest benefits in lowering both oxidative and inflammatory indices in PCOS subjects. Evidence from the current review showed an inconsistency with regard to the effect of Se on insulin resistance.

Overall, results of case-control studies suggest that patients with PCOS have lower serum levels of Se than control groups. Nonetheless, inconsistent relationships were observed between Se levels and reported PCOS complications $[13,18]$. Studies so far have not been able to find any significant correlation between serum levels of Se and insulin resistance $[13,17,18]$, However, Se levels are inversely associated with total testosterone [13]. In a study, Montes-Nieto et al. [14] indicated that both the distribution of body fat and the abundance of several VAT and SAT proteins including Se binding protein 1 (SBP1) and some antioxidant proteins are different between men and women. These patterns were similar in men and women with androgen excess, suggesting the potential effect of androgens on adipose tissue function, particularly gene expression of SBP1 which leads to reduced Se levels.

The results of an in vitro study support the antioxidant features of Se in the neutrophils of patients with PCOS [21]. Hyperglycemia induction in PCOS increases ROS production leading to systemic inflammatory response $[13,44]$. Particularly, the release of TNF- $\alpha$ following hyperglycemia exacerbates insulin resistance [13]. Elevated oxidative stress stimulates the ovarian steroidogenic enzymes responsible for androgen production causing hyperandrogenism [13].

Being widely used in inflammatory reproductive diseases, Se may inhibit NF- $\mathrm{k} \beta$ and reduce inflammatory cytokines including TNF- $\alpha$ and IL-1/18, thereby preventing insulin resistance $[21,45]$. It may be speculated that the systemic inflammatory condition in PCOS negatively influences Se concentration which results in exacerbation of inflammation [46-48]. Se acts as insulin-like agent and increases gene expression in pancreatic beta cells while downregulating COX-2 and P-selectin; therefore, treatment with Se may prevent diabetes [49-51].
In PCOS, neutrophils are the key immune cells producing inflammatory metabolites, especially interleukins and TNF- $\alpha[52,53]$. $\mathrm{Ca}^{2+}$ entry to the neutrophils through transient receptor potential (TRP) channels and the oxidative stress activate neutrophils leading to increased oxidative stress $[21,54,55]$. Köse et al. observed the modulatory effect of Se on TRPV1 channels which resulted in a considerable reduction in $\mathrm{Ca}^{2+}$ influx and antioxidant protection in the neutrophils isolated from PCOS patients [21]. A study by Kayan et al. indicated that $\mathrm{Se}$ inhibits $\mathrm{Ca}^{2+}$ influx through a TRP melastatin-2 (TRPM2) channel in hamster ovary cells in order to protect the cells against oxidative stress [56].

PCOS-induced Se deficiency inhibits PPAR-y activity involved in the metabolism of insulin as well as lipids and the regulation of ovarian function $[57,58]$. Se supplementation in infertile women with PCOS enhanced the gene expression of PPAR-y and GLUT-1, but did not affect LP (a) and downregulated LDLR leading to mitigation of metabolic disorders such as insulin resistance and dyslipidemia [16]. Previous studies have suggested that Se has possible therapeutic effects on hypercholesterolemia and hypertriglyceridemia mainly due to significant increase in the gene expression of very long chain dehydrogenase, medium chain acyl-CoA dehydrogenase, and $\beta$-oxidation enzymes $[59,60]$.

A study on 70 women with PCOS revealed that those received Se ( $200 \mu \mathrm{g}$ per day) or placebo for 8 weeks presented lowering effects of Se on serum insulin levels, HOMA-IR, serum triglycerides, and VLDL-C levels, however, FPG and other lipid profiles did not change [20]. Contrarily, Hosseinzadeh et al. observed no benefits of Se supplementation among 60 PCOS women who consumed Se or placebo with the same doses used in their previous study for 12 weeks [19]. Unexpectedly, it intensified the insulin resistance without significant changes in serum testosterone, SHBG, and free androgen index [19]. This study suggested that increased selenoprotein $P$ is responsible for insulin resistance following Se supplementation [61-65]. Selenoprotein $P$ inhibits a main regulator of energy metabolism, called adenosine monophosphate-activated protein kinase, and independently worsens insulin resistance [61]. Therefore, it can be hypothesized that the body is likely to compensate the metabolic disorders occurring in PCOS by reducing Se levels [19].

Oxidative stress is reported to induce both insulin resistance and hyperandrogenism [66]. On the other hand, hyperandrogenism, hyperinsulinemia, and insulin resistance in women with PCOS predispose the patient to infertility and hirsutism in addition to the mentioned complications [66-68]. Most included studies have conducted on Se and insulin resistance in PCOS [12,13,18-20], but there are few studies on the effect of Se on hormonal profile $[19,22]$. It has been shown that FSH tends to decrease in PCOS, however the concentrations of $\mathrm{LH}, \mathrm{E} 2$, testosterone, and DHEAS grow considerably [13]. LH and induced insulin resistance synergistically enhance the production of androgens in the thecal cells [69]. Further, a reduction in the hepatic synthesis of SHBG following Insulin resistance and hyperinsulinemia causes higher concentrations of free androgens $[69,70]$.

A novel Se-based immunomodulatory drug (IMOD) has exhibited protective effects against hyperandrogenism-induced PCOS [71]. This study attributed the positive effects of IMOD in part to the antioxidant and anti-inflammatory properties of Se [71]. Coksun et al. indicated a negative association between Se levels 


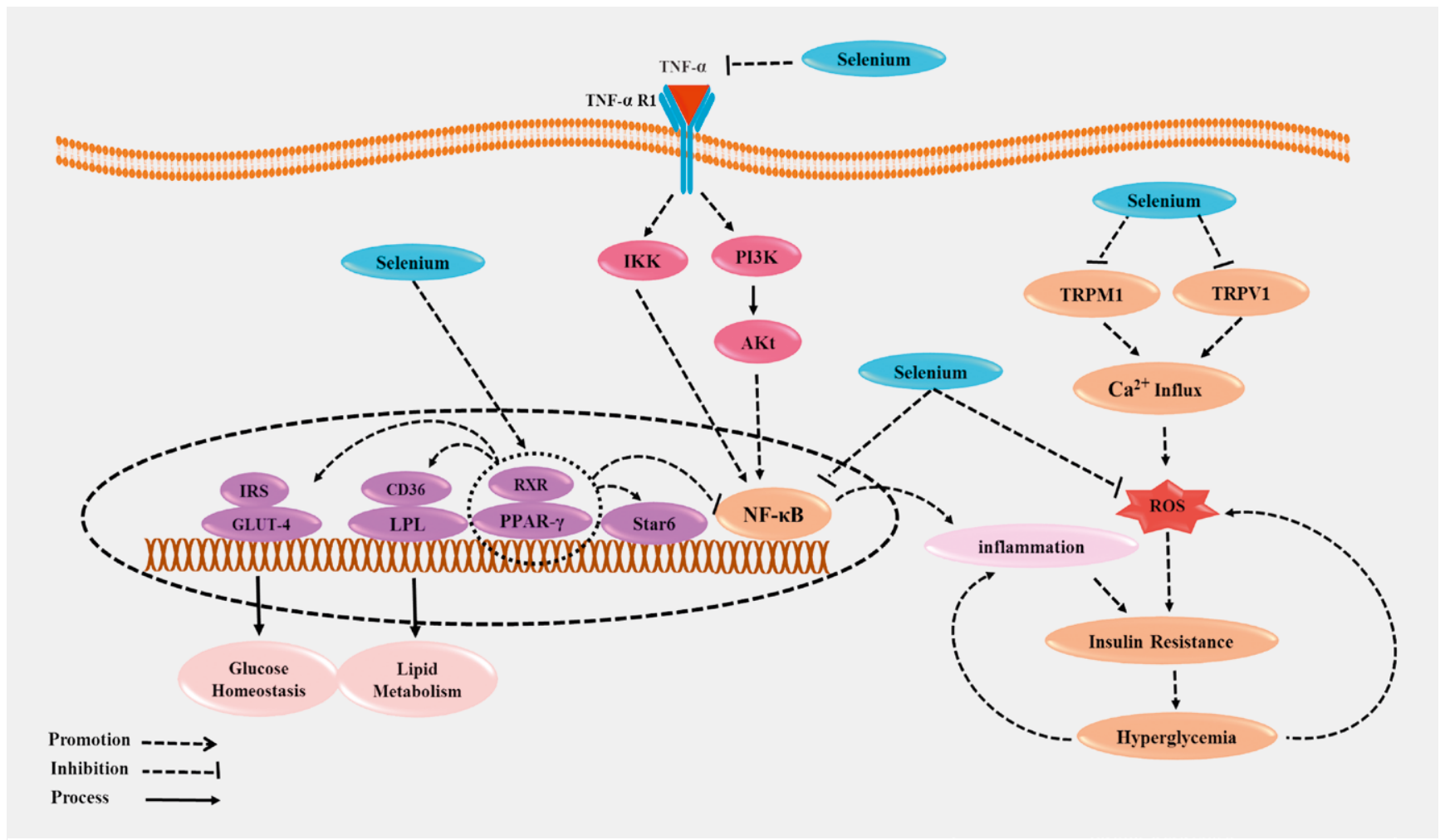

Fig. 3 Possible mechanisms for the impacts of selenium on polycystic ovary syndrome.

and $\mathrm{LH}$ and testosterone, although the authors could not demonstrate the causal relationship between Se depletion and hormonal disorders [13].

A randomized controlled trial amongst women with PCOS revealed significant positive outcomes including enhanced conception rate, reduced serum DHEA levels, hirsutism, alopecia, and acne in the Se supplemented group [22]. This study suggests that Se supplementation may exert its favorable effects on the hormonal profile through improving insulin metabolism and reducing oxidative stress [20,21]. As a whole, possible mechanisms for potential roles of Se on metabolic pathways in PCOS is summarized in > Fig. 3.

\section{Knowledge gaps and future directions}

None of the clinical trials has evaluated the effects of Se supplementation on plasma or urine Se levels at baseline and at the end of the study. Se intake may only be effective in patients with Se deficiency; thus, determination of Se status and a standard method for assessing its status should be considered in the studies. Future trials of Se supplementation across a large-scale population with optimal doses are required to confirm possible beneficial effects of Se in attenuation of PCOS complications, particularly insulin resistance, dyslipidemia, and hyperandrogenism. More studies are also warranted to address whether Se status contributes to PCOS pathogenesis or Se deficiency occurring due to dysregulated hormonal profile in PCOS.

Patients with PCOS consume various medications such as insulin sensitizers, anti-hyperlipidemic drugs and fertility medicines [72]. The interactions between Se and the relevant medications haven not been yet definitively assessed. Finally, considering the fact that oxidative stress may mediate hyperandrogenism in PCOS, the question arises whether reducing oxidative stress using any kind of antioxidants can mitigate hyperandrogenism or Se supplementation is capable to alter hormonal profile via exclusive mechanisms.

\section{Conclusion}

In conclusion, evidence from the reported studies confirmed the negative relationship between serum Se levels and some androgenic hormones in PCOS, although there are no robust documents to consider Se status as a direct contributor to PCOS pathogenesis. Interventional studies suggested that Se supplementation potentially confers additional benefits in patients with PCOS. Se possibly lowers oxidative stress leading to a significant reduction in insulin resistance and hyperandrogenism. Further, Se appears to attenuate dyslipidemia observed in PCOS through affecting gene expression involved in lipid metabolism. Taken together, the available data are currently insufficient to support the protective effects of Se on PCOS. Regarding the fact that there is a narrow window between the deficiency and toxicity of Se, the optimal dose of Se to mitigate the complications induced in PCOS is extremely important.

\section{Acknowledgment}

The authors thank the Student Research Committee, Tabriz University of Medical Science. 


\section{Conflict of Interest}

The authors declare that they have no conflict of interest.

\section{References}

[1] Shukla A, Mandel L. Polycystic Ovarian Syndrome. Obesity Management. Berlin: Springer; 2019: 31-40

[2] Azziz R, Carmina E, Chen Z et al. Polycystic ovary syndrome. Nat Rev Disease Primers 2016; 2: 16057

[3] Rosenfield RL, Ehrmann DA. The pathogenesis of polycystic ovary syndrome (PCOS): The hypothesis of PCOS as functional ovarian hyperandrogenism revisited. Endocr Rev 2016; 37: 467-520

[4] Rosenfield RL. Etiology and pathophysiology of polycystic ovary syndrome in adolescents. www.uptodate.com Wolters Kluwer

[5] Waldman IN, Legro RS. Polycystic ovary syndrome. In The Ovary. Amsterdam: Elsevier; 2019: 415-435

[6] Papalou O, MVictor V, Diamanti-Kandarakis E. Oxidative stress in polycystic ovary syndrome. Curr Pharm Design 2016; 22: 2709-2722

[7] Aquino Cl, Nori SL. Complementary therapy in polycystic ovary syndrome.Transl Med@ UniSa 2014; 9: 56

[8] Wrobel JK, Power R, Toborek M. Biological activity of selenium: Revisited. IUBMB Life 2016; 68: 97-105

[9] Kipp AP. Selenium and selenoproteins in redox signaling. Free Radical Biol Med 2017; 108: S1-S3

[10] Duntas LH, Hubalewska-Dydejczyk A. Selenium and Inflammation-Potential Use and Future Perspectives. US Endocrinol 2015; 11: 97-102

[11] Papalou O, Diamanti-Kandarakis E. The role of stress in PCOS. Exp Rev Endocrinol Metab 2017; 12: 87-95

[12] Shabani A, Noshadian M, Jamilian M et al. The effects of a novel combination of selenium and probiotic on weight loss, glycemic control and markers of cardio-metabolic risk in women with polycystic ovary syndrome. J Funct Foods 2018; 46: 329-334

[13] Coskun A, Arikan T, Kilinc M et al. Plasma selenium levels in Turkish women with polycystic ovary syndrome. Eur J Obstetr Gynecol Reprod Biol 2013; 168: 183-186

[14] Montes-Nieto R, Insenser M, Martínez-García MÁ et al. A nontargeted proteomic study of the influence of androgen excess on human visceral and subcutaneous adipose tissue proteomes. I Clin Endocrinol Metab 2013; 98: E576-E585

[15] Wickenheisser JK, Nelson-DeGrave VL, McAllister JM. Dysregulation of cytochrome P450 17 $\alpha$-hydroxylase messenger ribonucleic acid stability in theca cells isolated from women with polycystic ovary syndrome. J Clin Endocrinol Metab 2005; 90: 1720-1727

[16] Modarres SZ, Heidar Z, Foroozanfard F et al. The Effects of selenium supplementation on gene expression related to insulin and lipid in infertile polycystic ovary syndrome women candidate for in vitro fertilization: A randomized, double-blind, placebo-controlled trial. Biol Trace Element Res 2018; 183: 218-225

[17] Zagrodzki P, Krzyczkowska-Sendrakowska M, Nicol F et al. Selenium status parameters in patients with polycystic ovary syndrome. J Trace Element Med Biol 2017; 44: 241-246

[18] Kanafchian M, Mahjoub S, Esmaeilzadeh S et al. Status of serum selenium and zinc in patients with the polycystic ovary syndrome with and without insulin resistance. Middle East Fertil Soc J 2018; 23: 241-245

[19] Hosseinzadeh FM, Hosseinzadeh-Attar M], Yekaninejad MS et al. Effects of selenium supplementation on glucose homeostasis and free androgen index in women with polycystic ovary syndrome: A randomized, double blinded, placebo controlled clinical trial. J Trace Element Med Biol 2016; 34: 56-61
[20] Jamilian M, Razavi M, Fakhrie Kashan Z et al. Metabolic response to selenium supplementation in women with polycystic ovary syndrome: a randomized, double-blind, placebo-controlled trial. Clin Endocrinol 2015; 82: 885-891

[21] Köse SA, Nazıroğlu M. Selenium reduces oxidative stress and calcium entry through TRPV1 channels in the neutrophils of patients with polycystic ovary syndrome. Biol Trace Element Res 2014; 158: 136-142

[22] Razavi M, Jamilian M, Kashan ZF et al. Selenium supplementation and the effects on reproductive outcomes, biomarkers of inflammation, and oxidative stress in women with polycystic ovary syndrome. Horm Metab Res 2016; 48: 185-190

[23] Carmina E, Lobo RA. Polycystic ovary syndrome (PCOS): Arguably the most common endocrinopathy is associated with significant morbidity in women. J Clin Endocrinol Metab 1999; 84: 1897-1899

[24] Rotterdam ESHRE/ASRM-Sponsored PCOS Consensus Workshop Group. Revised 2003 consensus on diagnostic criteria and long-term health risks related to polycystic ovary syndrome. Fertil Steril 2004; 81: $19-25$

[25] Stranges S, Marshall JR, Natarajan R et al. Effects of long-term selenium supplementation on the incidence of type 2 diabetes: A randomized trial. Ann Intern Med 2007; 147: 217-223

[26] Carmina E, Lobo RA. Use of fasting blood to assess the prevalence of insulin resistance in women with polycystic ovary syndrome. Fertil Steril 2004; 82: 661-665

[27] Uno K, Katagiri H, Yamada T et al. Neuronal pathway from the liver modulates energy expenditure and systemic insulin sensitivity. Science 2006; 312: 1656-1659

[28] Liu M-L, Ylitalo K, Salonen R et al. Circulating oxidized low-density lipoprotein and its association with carotid intima-media thickness in asymptomatic members of familial combined hyperlipidemia families. Arterioscl Thromb Vascul Biol 2004; 24: 1492-1497

[29] Puttabyatappa M, VandeVoort CA, Chaffin CL. hCG-induced down-regulation of PPAR $\gamma$ and liver $X$ receptors promotes periovulatory progesterone synthesis by macaque granulosa cells. Endocrinology 2010; 151: 5865-5872

[30] Steinbrenner $\mathrm{H}$. Interference of selenium and selenoproteins with the insulin-regulated carbohydrate and lipid metabolism. Free Radical Biol Med 2013; 65: 1538-1547

[31] Apridonidze T, Essah PA, luorno M] et al. Prevalence and characteristics of the metabolic syndrome in women with polycystic ovary syndrome. J Clin Endocrinol Metab 2005; 90: 1929-1935

[32] Poretsky L. The insulin-related ovarian regulatory system in health and disease. Endocr Rev 1999; 20: 535-582

[33] Catteau-Jonard S, Dewailly D. Pathophysiology of polycystic ovary syndrome: the role of hyperandrogenism. Polycystic Ovary Syndrome. Delft: Karger Publishers; 2013: 22-27

[34] Jakimiuk AJ, Weitsman SR, Navab A et al. Luteinizing hormone receptor, steroidogenesis acute regulatory protein, and steroidogenic enzyme messenger ribonucleic acids are overexpressed in thecal and granulosa cells from polycystic ovaries. J Clin Endocrinol Metab 2001; 86: 1318-1323

[35] Macut D, Bjekić-Macut J, Savić-Radojević A. Dyslipidemia and oxidative stress in PCOS. Polycystic Ovary Syndrome.I Delft: Karger Publishers 2013; 51-63

[36] González F, Rote NS, Minium J et al. Reactive oxygen species-induced oxidative stress in the development of insulin resistance and hyperandrogenism in polycystic ovary syndrome. J Clin Endocrinol Metab 2006; 91: 336-340

[37] Mohamadin AM, Habib FA, Elahi TF. Serum paraoxonase 1 activity and oxidant/antioxidant status in Saudi women with polycystic ovary syndrome. Pathophysiology 2010; 17: 189-196 
[38] Fenkci IV, Serteser M, Fenkci S et al. Paraoxonase levels in women with polycystic ovary syndrome. J Reprod Med 2007; 52: 879-883

[39] Pawelczak M, Rosenthal J, Milla S et al. Evaluation of the pro-inflammatory cytokine tumor necrosis factor- $\alpha$ in adolescents with polycystic ovary syndrome. J Pediatr Adolesc Gynecol 2014; 27: 356-359

[40] Scicchitano P, Dentamaro I, Carbonara R et al. Cardiovascular risk in women with PCOS. Int J Endocrinol Metab 2012; 10: 611-618

[41] Steinbrenner H, Steinbrenner H, Bilgic E et al. Selenoprotein P protects endothelial cells from oxidative damage by stimulation of glutathione peroxidase expression and activity. Free Radical Res 2006; 40: 936-943

[42] Zou X-F, Ji Y-T, Gao G et al. A novel selenium-and copper-containing peptide with both superoxide dismutase and glutathione peroxidase activities. J Microbiol Biotechnol 2010; 20: 88-93

[43] Zeng J, Zhou J, Huang K. Effect of selenium on pancreatic proinflammatory cytokines in streptozotocin-induced diabetic mice. J Nutr Biochem 2009; 20: 530-536

[44] González F, Rote NS, Minium J et al. Reactive oxygen species-induced oxidative stress in the development of insulin resistance and hyperandrogenism in polycystic ovary syndrome. J Clin Endocrinol Metab 2006; 91: 336-340

[45] Wang $Y$, Lin M, Gao $X$ et al. High dietary selenium intake is associated with less insulin resistance in the Newfoundland population. PloS One 2017; 12: e0174149

[46] Hesse-Bahr K, Dreher I, Kohrle J. The influence of the cytokines II-1beta and INFgamma on the expression of selenoproteins in the human hepatocarcinoma cell line HepG2. Biofactors 2000; 11: 83-85

[47] Nichol C, Herdman J, Sattar $\mathrm{N}$ et al. Changes in the concentrations of plasma selenium and selenoproteins after minor elective surgery: Further evidence for a negative acute phase response? Clin Chem 1998; 44: 1764-1766

[48] Renko K, Hofmann PJ, Stoedter M et al. Down-regulation of the hepatic selenoprotein biosynthesis machinery impairs selenium metabolism during the acute phase response in mice. FASEB J 2009; 23: $1758-1765$

[49] Li YB, Han JY, Jiang W et al. Selenium inhibits high glucose-induced cyclooxygenase-2 and P-selectin expression in vascular endothelial cells. Mol Biol Rep 2011; 38: 2301-2306

[50] Stapleton SR. Selenium: an insulin-mimetic. Cell Mol Life Sci 2000; 57: 1874-1879

[51] Hei Y], Farahbakhshian S, Chen X et al. Stimulation of MAP kinase and S6 kinase by vanadium and selenium in rat adipocytes. Mol Cell Biochem 1998; 178: 367-375

[52] Victor VM, Rocha M, Banuls C et al. Induction of oxidative stress and human leukocyte/endothelial cell interactions in polycystic ovary syndrome patients with insulin resistance. J Clin Endocrinol Metab 2011; 96: 3115-3122

[53] Victor VM, Rocha M, Banuls C et al. Mitochondrial complex I impairment in leukocytes from polycystic ovary syndrome patients with insulin resistance. J Clin Endocrinol Metab 2009; 94: 3505-3512

[54] Ayub K, Hallett MB. Ca2 + influx shutdown during neutrophil apoptosis: Importance and possible mechanism. Immunology 2004; 111: 8-12

[55] Naziroglu M, Sahin M, Cig B et al. Hypericum perforatum modulates apoptosis and calcium mobilization through voltage-gated and TRPM2 calcium channels in neutrophil of patients with Behcet's disease. J Memb Biol 2014; 247: 253-262

[56] Kayan M, Naziroglu M, Ovey IS et al. Non-ionic contrast media induces oxidative stress and apoptosis through $\mathrm{Ca}(2)(+)$ influx in human neutrophils. J Memb Biol 2012; 245: 833-840
[57] Uno K, Katagiri H, Yamada T et al. Neuronal pathway from the liver modulates energy expenditure and systemic insulin sensitivity. Science 2006; 312: 1656-1659

[58] Puttabyatappa M, Vandevoort CA, Chaffin CL. hCG-induced down-regulation of PPARgamma and liver $\mathrm{X}$ receptors promotes periovulatory progesterone synthesis by macaque granulosa cells. Endocrinology 2010; 151: 5865-5872

[59] Kim JE, Choi SI, Lee HR et al. Selenium significantly inhibits adipocyte hypertrophy and abdominal fat accumulation in OLETF rats via induction of fatty acid beta-oxidation. Biol Trace Elem Res 2012; 150: 360-370

[60] Kaur HD, Bansal MP. Studies on HDL associated enzymes under experimental hypercholesterolemia: Possible modulation on selenium supplementation. Lipids Health Disease 2009; 8: 55

[61] Misu H, Takamura T, Takayama $\mathrm{H}$ et al. A liver-derived secretory protein, selenoprotein P, causes insulin resistance. Cell Metab 2010; 12: 483-495

[62] Yang S], Hwang SY, Choi HY et al. Serum selenoprotein P levels in patients with type 2 diabetes and prediabetes: Implications for insulin resistance, inflammation, and atherosclerosis. J Clin Endocrinol Metab 2011; 96: E1325-E1329

[63] Roman M, Lapolla A, Jitaru P et al. Plasma selenoproteins concentrations in type 2 diabetes mellitus - a pilot study. Transl Res 2010; 156: 242-250

[64] Hurst R, Armah CN, Dainty JR et al. Establishing optimal selenium status: Results of a randomized, double-blind, placebo-controlled trial. Am J Clin Nutr 2010; 91: 923-931

[65] Xia Y, Hill KE, Li P et al. Optimization of selenoprotein P and other plasma selenium biomarkers for the assessment of the selenium nutritional requirement: A placebo-controlled, double-blind study of selenomethionine supplementation in selenium-deficient Chinese subjects. Am J Clin Nutr 2010; 92: 525-531

[66] Suresh S, Vijayakumar T. Correlations of insulin resistance and serum testosterone levels with LH:FSH ratio and oxidative stress in women with functional ovarian hyperandrogenism. Indian J Clin Biochem 2015; 30: 345-350

[67] Hauner H, Ditschuneit HH, Pal SB et al. Fat distribution, endocrine and metabolic profile in obese women with and without hirsutism. Metabolism 1988; 37: 281-286

[68] Munzker ], Hofer D, Trummer C et al. Testosterone to dihydrotestosterone ratio as a new biomarker for an adverse metabolic phenotype in the polycystic ovary syndrome. J Clin Endocrinol Metab 2015; 100: 653-660

[69] Poretsky L, Cataldo NA, Rosenwaks Z et al. The insulin-related ovarian regulatory system in health and disease. Endocr Rev 1999; 20 : 535-582

[70] Pugeat M, Crave JC, Elmidani M et al. Pathophysiology of sex hormone binding globulin (SHBG): relation to insulin. J Steroid Biochem Mol Biol 1991; 40: 841-849

[71] Rezvanfar MA, Rezvanfar MA, Ahmadi A et al. Molecular mechanisms of a novel selenium-based complementary medicine which confers protection against hyperandrogenism-induced polycystic ovary. Theriogenology 2012; 78: 620-631

[72] Teede H], Misso ML, Costello MF et al. Recommendations from the international evidence-based guideline for the assessment and management of polycystic ovary syndrome. Human Reprod 2018; 33: $1602-1618$ 